\title{
Socially-Oriented Requirements Engineering - Software Engineering Meets Ethnography
}

\author{
Sonja Pedell ${ }^{1,3}$, Tim Miller ${ }^{2}$, Frank Vetere ${ }^{1}$, Leon Sterling ${ }^{3}$, Steve Howard ${ }^{1}$ \\ ${ }^{1}$ Department of Information Systems, The University of Melbourne \\ ${ }^{2}$ Department of Computer Science and Software Engineering, The University of Melbourne \\ 111, Barry Street, 3010 Carlton, Victoria, Australia \\ \{pedells, tmiller, fv, showard\}@unimelb.edu.au \\ ${ }^{3}$ Faculty of Information and Communication Technologies, \\ Swinburne University of Technology \\ 3122 Hawthorn, Victoria, Australia \\ \{lsterling\}@swin.edu.au
}

\begin{abstract}
We outline an approach for eliciting, understanding, and representing the cultural aspects of the domestic environment for the purpose of system design. We use agent models as shared artefacts to represent the everyday cultural life of the home. These representations build an understanding between the people that own this culture and the people responsible for technology development. We argue the necessity of knowing about a formal representation of these cultural aspects to inform design decisions and develop technologies that truly satisfy and support the everyday life of families. Our aim is to express socially-oriented requirements for technology. We show the usefulness of this approach on a case study that investigates interactions between grandparents and grandchildren who are geographically separated.
\end{abstract}

Keywords: Socially-oriented requirements, shared artefacts, cultural design, intergenerational interactions.

\section{Introduction}

Technology can facilitate interpersonal contact in social interactions, but only if it addresses and fulfils the felt needs of people acting in their social contexts. The felt needs include those that are emotional or behavioural, such as experiencing playfulness, feeling engaged, or being capable of expressing intimacy [21, 27, 40, 43, 44]. Such socially-oriented requirements are important to human culture but are difficult to specify and measure. Consequently, engineering systems to fulfil them is a non-trivial task. The functionality needed to facilitate a socially-oriented requirement is often unclear; for example, how do we engineer a system to ensure it is fun?

Ethnographic data can be used to inform system models and to help define socially-oriented requirements [30, 42]. However, ethnographic data does not translate into requirements in a straightforward manner. Themes extracted from ethnographic data are not functional requirements [36]. Ethnographies are rich 
descriptions of human activities and cultural practices, and do not define the behaviour of of technological systems. Ethnographic data tends to be a bottom-up view of the domain, while system models are typically derived top-down. Informing system models with ethnographic remains challenging.

This chapter defines a method for addressing the gap between ethnographic data and system models created with agent-oriented techniques. We argue that the agent paradigm [39] is suitable for modelling the social domain because it allows representation of the goals and motivations of agent roles and individuals. By social domain, we mean those practices that encompass cultural activities and embrace shared values. Specifically, we argue quality goals can be used to discuss sociallyoriented requirements such as having fun and being playful. Our method substantiates and refines agent-oriented quality goals with attributes and new understandings about domestic cultural practices obtained from the ethnographic data.

In our method, software developers firstly define a high-level goal model that includes quality goals relevant for a specific cultural context, such as "show affection". Ethnographic practices are then followed to obtain data about the particular domain and its value-based activities. The goal models are used as a conceptual lens through which the ethnographic data is analysed from a cultural point of view. From the data, themes are discerned, and where appropriate, a theme is attributed to a high-level quality goal. If a theme does not correspond to a quality goal, this triggers a discussion as to whether a new quality goal is required. The result is an agent model with concrete themes that exemplify how the quality goal can be fulfilled.

The domain focus of our research is the everyday life of people at home. The problem focus is how to develop technology to support interactions between family members when they are separated from each other, such as grandparents \& grandchildren, intimate partners, and elderly people who are isolated from family members and friends. Thus, we are modelling human motivations and contributions to it through everyday culture. Culture here does not refer to the particular ethnic aspects that characterize it, but rather the culture of everyday interactions between family and friends. To build systems that are sensitive to this culture, we represent the everyday in terms of small, mundane yet meaningful interactions [21, 40]. Technologies for strengthening bonds within separated families must fulfil hard-to define goals such as showing presence and engaging over distance.

Our particular study examines technology for supporting the relationship between grandparents and grandchildren who are geographically separated. This study presents many interesting and challenging problems for defining innovative technologies that integrate within existing cultures. To gather field data, we use cultural probes [13] and more specifically technology probes [22] for generating insights into the interactions between grandparents and their grandchildren. The agent models are used in multiple ways throughout the development process. They serve:

- to representing an understanding of intergenerational interaction.

- as a conceptual lens through which we analyse collected field data leading to an evaluation of the original model. 
- as a shared artefact between the grandparents and grandchildren, and the software engineers and ethnographers, in order to understand the cultural aspects of the home.

The approach of interleaving agent models and simple technologies helps us to improve our understanding of grandparent-grandchildren interactions and addresses the gap between ethnographic data and system models as it provides better models that are substantiated by human practice. The chapter aims to:

- increase the modelling capability of social domains using agent-oriented concepts

- understand goals modelled using agent-oriented techniques, and their associated qualities, in the light of technology use over a distance, and

- provide a method for designing and implementing non-standard quality requirements within complex social settings, such as the domestic space.

\section{Designing for the Home}

There has been a growing interest in recent years in designing domestic technologies, in particular in supporting family interactions in the home and across homes $[5,15$, $24,35,43,44,45]$. Domestic design has evolved from effective functional and smart technologies [18] to more subtle and less purposeful ones, such as those for digitally mediated relationships [15] and lightweight communication [29]. Domestic technologies for connecting family members tend to be less concerned with informational needs, and dedicated more to connecting families in their specific and often diffuse ways, such as passing the time together [21]. Recent research in domestic technology includes designing phatic technologies [41] for e.g. mediating intimacy between couples [14], connecting distant family members [e.g. 24, 43] and connecting older people $[6,28]$. We consider the home as a domain where culture is lived and fostered. Family life is an important carrier of our cultural life; the space where traditions are passed on. How do we develop and evaluate technologies mediating such subtle meanings such as spending time together that often only become apparent over the long-term or in hindsight? Currently, there is no comprehensive means of deriving culturally shaped social needs for informing agent based quality goals.

The development of domestic technologies commonly presented in the literature is often based on field data collected in the home. Research about the contact between family members is frequently explored by introducing custom-made technology into homes and by interviewing inhabitants to investigate the success of the introduced technology [24, 29, 443, 44]. In most cases, little information is given on how designers progress from social needs to domestic technology, and how well the technology fulfils the family needs. Baxter and Sommerville [2] suggest that it is not enough to analyse the situation from a socio-technical perspective and then explain the analysis to engineers. According to them approaches are needed that are pragmatic and use terminology not alien to engineers. We contribute to this work by 
exploring the relationship between complex value laden interactions and functional richness in domestic settings, as captured and communicated through agent-oriented models.

Getting from data of domestic lives and routines to useful and suitable technologies for the inhabitants of the home and their family relationships presents many challenges for ethnographers and software engineers. One of the big challenges for domestic design is that there is no such thing as a 'typical home' [45] or clear set tasks. Leonardi et al. [27, p.1703] describe the home as " $a$ 'territory of meaning', a place where pleasure, affect and aesthetics are deeply interwoven with the functional and utilitarian dimensions." There is a gap between the design of domestic technologies and our understanding of the inhabitant's needs, as inhabitants represent a diverse population with non-functional and often ambiguous needs and desires that are not easy to articulate [21].

Home is a special place and designing for the home requires approaches different to traditional ones [3]. In order to communicate effectively, domestically focussed design teams need a shared language, which is sensitive to their specific practices [8]. Field researchers facilitating participatory design activities, and technology developers responsible for interpreting the designs for actual technologies, share the purpose of creating human-oriented technology but face very different challenges. Software engineers usually focus on future technologies and social needs are often neglected in development practice [38]. The ethnographers focus is on the current lives of people. Consequently there are gaps and disconnections that both professional groups have to bridge in the design process. We aim to address this gap with the help of agent-based motivational goal models used to understand and build culturallysensitive technologies.

In our case study, we are concerned with a particular type of social goal - the goal of having fun. Having fun is not simply a matter of creating a game or providing a range of communication channels. Fun is more elusive and can be subtle in its manifestations. Fun is not typically embodied in functional aims, but is expressed via social values such as simply spending time with each other. Fun comes in many forms and there are a myriad of possibilities of how fun can be realised. Research about such positive emotions around technology use is becoming increasingly important [19]. Fun and enjoyment are as important in the home as productivity and efficiency are in the work context. In order to create fun oriented domestic technologies, we need tools that are able to carry the complex, abstract and often ambiguous insights of field data collected from family cultures into the development process.

\section{The Culture of Family Life}

Other authors have focused on cultural aspects in the home and focus on the values of family members [4]. The home is a space where culture is learnt, passed on and lived. Values are expressed via activities that are embedded in daily life. We are interested in exploring the social goal having fun. In this regard we look into the cultural aspects 
of one domain - the family home. We see culture as an influence over and outcome of family life.

Specifically we investigate fun resulting from intergenerational interactions and how these can be mediated. Several research projects have dealt with the grandparentgrandchild relationship and its technology support over a distance [e.g. 9, 10, 29, 43, ] covering a wide range of interactions such as 'telling mobile stories' [9], 'sharing a photo frame' [10] and 'using a lightweight messaging device' [29].

The grandparent-grandchild relationship is an example of a set of complex social interactions. This relationship plays an important role in our culture as the interactions between generations leads to an exchange of traditions and values. As such grandparents and grandchildren are not users in the traditional sense, but inhabitants of their particular social world with their own routines and personal lifestyles. They are living with complexities: grandparents have to fulfil a wide range of ill-defined roles to live up to being grandparents and more so being grandparents to have fun with. Interactions are based on subtle, underlying values. These values are part of the intergenerational relationship. It is a challenge to support them adequately with a suitable range of functionalities when families are separated by distance and the faceto-face exchange is reduced. Therefore, we have to look at a family's values more closely to understand emerging interactions in technology use. We analyse such interactions in the light of these values in order to draw conclusions about the nature of domestic technology. Our models help us make pressing function allocation decisions: which roles can be taken on by software agents to support culture, and which should remain with people (human agents).

\section{Socially-Oriented Requirements Engineering with Agent Models}

Typical goals in socially-oriented systems are ambiguous, non-instrumental, subtle and long term [31], and are difficult to describe and account for in ways that are appropriate for technology development. Established development tools typically deal best with clearly defined, hierarchical goals that endure over a specified time frame. Some domestic and social goals are difficult to capture with these tools. Pavon et al. [33] argue that agent-based models are ideal for understanding the complex topics inherent in human organisations because the concepts used in these models are suitable for expressing the behavioural aspects of individuals and their interactions. We use agent modelling to represent goals and interactions, such as culturally defined roles played by the different stakeholders in the system, the related goals of the different stakeholders/roles, and the relationships between these [39].

The value of matching socially-oriented studies of human interaction with user requirements elicitation methods in order to abstract activity and embed technology into social contexts has been acknowledged [e.g. 42]. Other researchers describe bridging the gap between the output of field studies and the required input to system development through meta-modelling [23]. However this is not straightforward for socially-oriented requirements. Eliciting socially-oriented requirements from field 
data involves working in a milieu in which it is essential to capture concepts accurately but flexibly and at a high level, without losing the liveliness and vitality of those concepts through overly detailed specification. For example, the role of a grandparent does not come with an established list of responsibilities or a minimum performance plan. The roles that grandparents see themselves playing in the life of their grandchildren are highly dependent on many factors such as the individual, their experience with their own grandparents, and their cultural background.

In our approach we use the following main components:

1. Starting with motivational models with a focus on quality goals.

2. Implementing lean, but focused technologies.

3. Lightweight evaluation of quality goals using ethnographic studies.

4. Substantiating quality attributes of use activities in quality clouds.

5. Iterative exploration and discussion of social requirements.

6. Refining of user needs for domestic technologies.

In this paper, we present the components of the method and not the overall process. The activities of these components take place iteratively, depending on the available knowledge of the user domain.

\subsection{Motivation Models}

Here we build further on the work of Sterling and Taveter [39]. Their research has focused on how to make high-level agent-oriented models palatable in design discussions. This is achieved by using goal models that have a straightforward and easy syntax and semantics. Goal models are useful at early stages of requirements analysis to arrive at a shared understanding [17, 25]; and the agent metaphor is useful as it is able to represent the concepts that we want to capture for socially-oriented systems, such as agents (people) taking on roles associated with goals. These goals include quality attributes that are represented in a high-level pictorial form and that are used to inform and gather input from stakeholders. In Sterling and Taveter's notation, goals are represented as parallelograms, quality goals are clouds, and roles are stick figures. These constructs are connected using arcs, which indicate relationships between them.

We started with a set of high-level qualities or values of grandparent-grandchild interactions: share fun, show presence, and show affection (component 1). Activities that would support these specific aspects of intergenerational interactions are gifting, playing, and show \& tell. These values and abstract activities are represented in a high-level goal model (see Figure 1). The goals depicted as parallelograms represent meaningful activities in the grandparent-grandchild interaction. The quality goals represented by clouds are high-level attributes that are often subjective, contextspecific, and imprecise, and are therefore able to express nature of intergenerational fun. We included such quality goals as part of the design discussions since they accounted for social values embedded in intergenerational fun interactions. The agent-oriented model of Intergenerational Fun depicted in Figure 1 served as a 
common basis for design discussions of building three technologies by three different design teams - one of the technologies discussed in detail in the next section.

Quality requirements at the early stages of elicitation tend to be imprecise, subjective, idealistic and context-specific [25]. Garcia and Medinilla [11] describe high-level quality goals as a specific form of uncertainty that can be used as a descriptive complexity reduction mechanism and to model and discuss uncertainties in the environment. In our requirements elicitation process, we seek complexity reduction without losing the richness of the social concepts themselves. Instead of eliminating uncertainty early in the process, we embrace it and withhold design commitment, at least until there is clarity and understanding [12]. High-level goals associated with activities can act as a point of reference for discussing the usefulness of design alternatives to achieve these goals instead of decomposition into single requirements.

\subsection{Quality Goals}

Focussing on quality is well established within software and systems engineering. Software engineers are aware of the need to express quality attributes of software as well as functional capabilities of software. These quality attributes are referred to using a variety of terms including: non-functional requirements, constraints, quality attributes, quality goals, soft goals or quality of service requirements [16, 26, 37, 38]. We use the construct of quality goals attached to functional goals to represent quality attributes of social interactions. Quality goals are essentially non-functional and are designed to encapsulate social aspects of the context into the software requirements model, thus providing a mechanism to carry subtle nuances of those social aspects through to the implementation phase. These quality goals remain interpretably flexible, even until the final product, opening up a variety of possible interpretations both in the design and use of the system. Some of the goals might influence the choice of functions and some might remain with the human agents - in our study, the grandparents and grandchildren. There is benefit in articulating socially-oriented quality goals without the need to resolve them into measurable goals or requirements that are implementable. We introduce culturally-influenced qualities as part of a socio-technical system tied to the motivations of their users. Sterling and Taveter's agent-oriented models allow the expression of non-functional requirements by attaching quality goals to goal models [39]. In our approach there is a direct pairing between system goals and quality goals, whereas non-functional goals do not generally have a direct relationship with functional goals [7]. This makes it more difficult to carry them through the process in an unresolved state. Relating an abstract and unresolved quality attribute to a system goal enables a focus on social goals throughout the design process. 


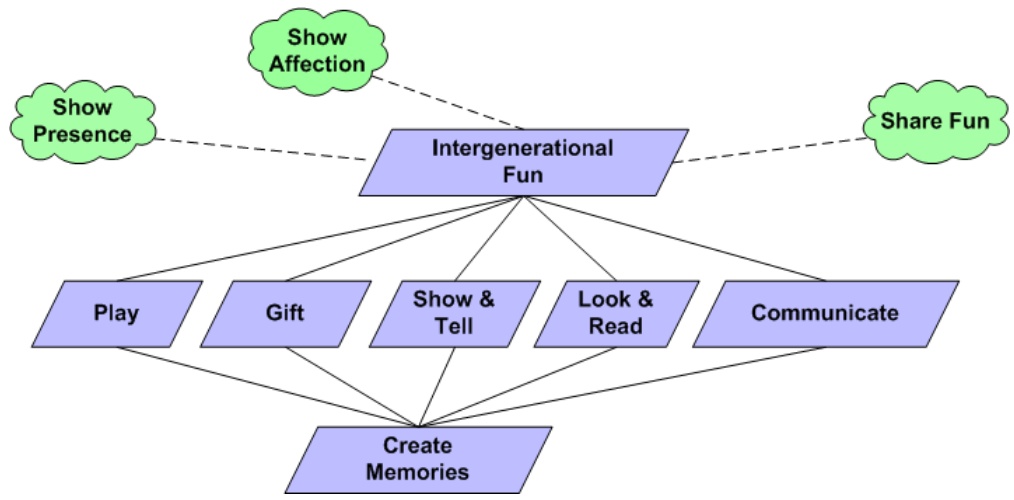

Fig 1. Agent-oriented model representing intergenerational fun.

From a software engineering point of view, the models enable us to take the outputs from a field study and use them to inform system development. This is achieved by taking account of the richness of human social interaction provided by the probe data, encapsulating quality attributes of that interaction into quality goals in the models, and using these models as inputs to the design process.

\section{Using Technology Probes to Obtain Cultural Data}

In our case study we built three technology probes that were inspired by the motivational model from Figure 1; Collage, electronic Magic Box, and Storytelling. The technologies were seen as instances of the goal model and emphasise various goals of the model. While Collage has its focus on 'playing' and Storytelling on 'show and tell' activities, the electronic Magic Box has its focus on 'gifting'. Next we describe the results and the procedure for informing quality goals focussing on the electronic Magic Box.

\subsection{Benefits of Probes in Developing Domestic Technologies}

Probes [13] are particularly suited to investigating people's everyday life in situations difficult to reach with traditional social science methods, such as questionnaires, interviews, focus groups or participant-observation. Rather than relying on the presence and intervention of the researcher, probes are designed to encourage and empower subjects to collect data themselves [1]. The participants use the probes to provide some insight, at their discretion, about their daily lives. Probes are specifically suitable for collecting data in the domestic domain through their ability to capture the nuanced aspects of everyday life $[1,20]$. Information and story generation are two important benefits that we see in the use of probes. Our approach required minimal ongoing intervention from the researchers, while allowing observation of the 
transactions between the participants. Therefore, the three technologies had logging capabilities to monitor and record the use of the applications serving as technology probes [22]. The interactions of all three systems, the messages and photographs were saved on different servers.

\subsection{Benefits of Goal Models}

The agent-oriented models are particularly suitable to be combined with technology probes in field studies. Firstly, we see agent-oriented models as a suitable way to express field data. As data gathered using probes are intentionally fragmentary and unstructured, the process of translation from field data to the abstract generalisation required in development is problematic. A process of combining technology probe data collection and agent-oriented models allows us to talk about intangible outcomes; such as that arising from fieldwork which can be surprising, complex, but subtle. The agent-oriented models provide a place where abstract design concepts can be collected and represented [34]. They are a lens through which use activities can be analysed and recorded and then discussed among researchers and software engineers. Secondly, agent-oriented models are part of a development methodology and can be combined with motivational scenarios, roles and domain models [39], each of them describing and providing context of the domain, which is important because contextual information offered by technology probes is often lost after data analysis.

\subsection{Technology Set Up}

The technology probes (component 2) used synchronous touch screens for display, and mobile camera phones for sending photographs and messages that were shared amongst the grandchildren and grandparents households. Each household was allocated one mobile phone and one touch screen; that is the grandparent household shared one phone and one touch screen between them and the grandchildren and parents shared one phone and one touch screen between them.
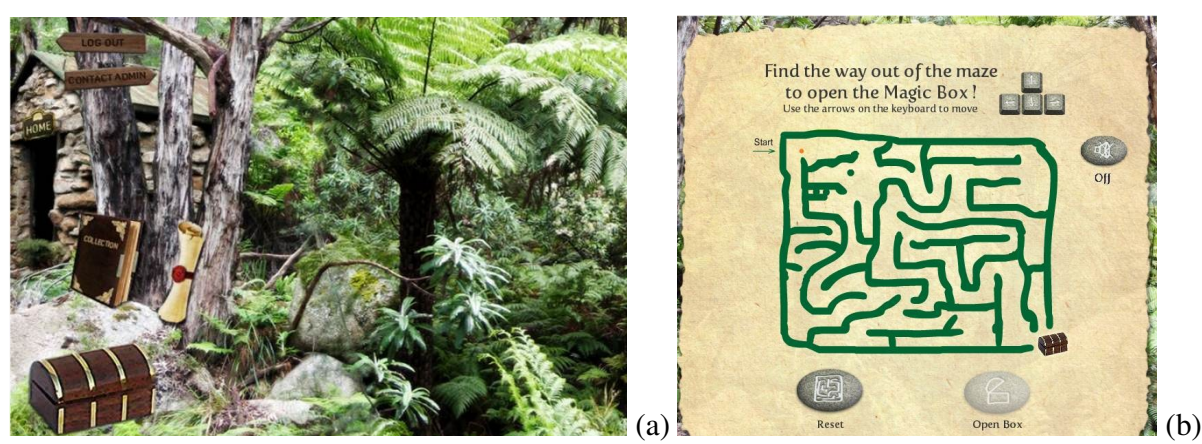

Fig. 2. Electronic Magic Box. A treasure box filled with a message (a) doing the maze (b) 
The mobile phones were important as we wanted sharing of everyday experiences. Grandparents and grandchildren could carry the phones with them and share photographs of events and ideas with the others sending it to the system without the need to be home. We chose technologies that constrained use as little as possible thereby facilitating flexible interactions without strict assumptions about how the technology was to be used. The systems were placed in high traffic areas in the family homes (e.g. lounge room or kitchen). While our focus was on the grandparentgrandchild relationship, the parents took on an important role in facilitating interactions and observing them without being active users.

The electronic Magic Box, a technology probe described here, allowed the sending of a treasure box that could be filled with photographs and messages. Figure 2a shows the layout of the opening screen. On the left side, seven picture-based links (home, magic box, scroll, collection book, settings, admin, and logout) can be found that guide the user to a number of destinations within the application. The box is placed in a forest of fern trees and appears either closed (a new box has arrived) or open (no new box has arrived). A scroll either sealed or with a broken seal indicates if the box in the other household has been opened and the content been looked at. In order to be able to access the content the receiver has to play a maze game (Figure 2b) to 'find' and open the box. An opened message can be saved in a collection book. Emphasis in this application was placed on the goal 'gifting', but the concept certainly carried elements of and was inspired by the other high-level goals of playing, show \& tell, look \& listen, communicating and creating memories.

\subsection{Study Design and Participants}

We introduced the three probe technologies to three families. The applications were installed in the family homes for three to six weeks over an elapsed period of four months (component 3). The children were aged between 5 and 9 years and the grandparents lived between 8 and 16 kilometres away from the children. All grandparents had regular contact with their grandchildren and all described having a strong and loving relationship.

\subsection{Data Collected}

We conducted three to four interviews per household about the probe use (usually grandparent and parent/grandchild interviews were conducted separately) - in total twenty interviews were conducted. The parents' presence in the grandchildren interviews was an important source of information as they were observing the ongoing interactions without being active users and were able to comment on changes since introduction of the technology probes. During the interviews we did not specifically ask questions about playing or gifting. These goals are implicit in the system as we tried to provide activities that supported these goals. We were more interested in the social interactions and how the qualities were judged by the 
participants. For example we would ask: "what kind of interactions did the system support?" and "what activities did you particularly enjoy?". If we did not obtain feedback that using the technology was fun, then we would have felt our original model was invalidated. The technology probe data collected with the electronic Magic Box consisted of 102 boxes (electronic letters and photographs), meta data about each box such as send times, and data from seven interviews.

\section{Analysing and Discussing Cultural Data}

The success of a design in achieving its goals can really only be investigated after implementation. The technology probes embodied certain goals of the goal model. We purposely kept the goals at a high level that was representative and comprehensive, as determined by the development team, but independent of any future implementation. Therefore, we were able to link the qualities learned during the ethnographic studies to the motivational models. The transcribed interviews together with photographs and electronic letters were analysed using content analysis [32]. The quality goals played the role of overarching themes for analysis. We explored intergenerational activities and interactions rather than technology per se. We were able to find sub-themes for all of the quality goals and therefore to learn more about each goal in the light of typical social activities between grandparents and grandchildren (component 4). Each sub-theme was briefly described and substantiated by compelling examples and instances of these goals in the context of intergenerational fun during use.

We analysed the interview data according to what we could learn about the quality goals, using the model in Figure 1 as an interpretive lens. The photographs and messages were analysed and discussed at regular project meetings. The essence of the quality goals was based on experiences and judgement of the participants regarding their interactions. Therefore, the interview data played a major role in this analysis as we wanted to expand from the activities and original goals to inform the quality goals. The photographs and messages were used mostly to confirm and illustrate the results with particular episodes and participant stories. The analysis helped us to keep the focus on the human needs with the technology as mediator. The purpose of the technology was not just to support intergenerational fun, but to support the development team in further investigating the qualities of the social goals in the model themselves.

The sub-themes that emerged from our data analysis were organised as characteristics to the quality goals into so-called quality clouds (Figure 3 ). The quality clouds consist of one quality goal - still linked to a functional goal - with associated qualities factored around. The quality clouds can be seen as an abstract representation of field data into which we are able to zoom into the associated quality goal more closely. The quality cloud shown in Figure 3 concerns the quality goal 'show affection' and its associated quality attributes. In this process the sub-qualities or 
quality attributes were formulated into adjectives to re-connect the qualities in discussions more easily to the functional goals they are attached to.

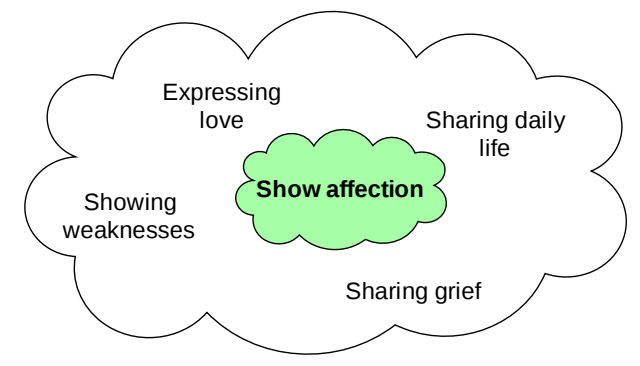

Fig. 3. Resulting quality cloud of analysed quality goal show affection

Each sub-quality of a main quality goal is briefly described and directly linked to the respective quotations in the interview data. In that regard the quality goals were augmented by ethnographic data. While we were interested to group the sub-qualities to our existing quality goals, in order to substantiate them with our field data, we permitted new main quality goals to emerge from the ethnographic data, and hence allow changes to our overall goal model. As part of the method, in the event important activities or themes evolve for which we cannot find a home, we define new quality goals.

\section{Results}

\subsection{Substantiating Quality Goals - Show Affection}

Below we present a sample of the interview data that demonstrates the process for elaborating the quality cloud show affection. Some of the sub-qualities from the clouds that brought us unexpected insights are described in more detail.

Share daily life: The aim was to share with and include the other family member in the happenings of the own life.

A. (granddaughter) tells nanna everything! For example the photo of the 'Dog sick'! - anything that took her interest on that particular day. That particular hour - absolutely everything she wants to tell her nanna [parent].

Show weaknesses: Family members were comfortable not only showing their best side, but also failures and weak points, because there is a loving trust within the relationship.

A challenge for most of the grandparents was managing the technology. Uncovering this kind of "weakness" is a very intimate act in itself. Problems dealing with the electronic Magic Box were often communicated in a humorous way or 
loaded with self-irony making the technology handling a shared episode in itself. A nice example was one grandmother sending a picture of her granddaughter along with this message:

Dear Andrea, in trying to send this photo to you I burnt my steak I am having for dinner, yuk!!! After this she took a photo of her burned frying pan as well and sent it (Figure 4a): When I tried to send this message Thursday the machine told me to try again, so here I am. This is the pan I burned while trying to enter the project!!!
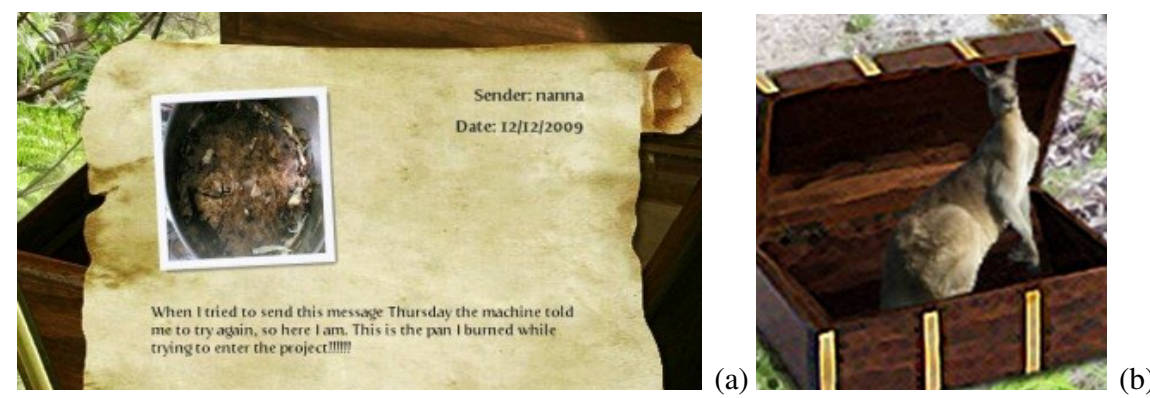

Fig. 4. The burnt steak (a) and the surprise kangaroo (b).

Our families tended to show themselves to people they trust and love. This grandmother assured the researchers that the pan was "all clean again" and that she had no more disasters. In a similar way one grandmother sent a photograph of her messy desk.

"This is my messy desk. I am trying to catch up with office work". The granddaughter took it up immediately as something funny and kept saying in the interviews: "Granny you are messy as well - you sent me this photograph of your desk."

That the grandparents admitted to weaknesses being adults and "should know better" was received as something very special by the grandchildren.

Express love: It was very common to send a message that explicitly expressed love or physical closeness.

"I love you" - messages and the building of a little sculpture with a sign "Nana gives the best hugs" sent as photograph are examples.

Share grief: The electronic Magic Box was particularly well suited in mediated shared emotions. There was sometimes an urge to transfer something important and emotional. One example was when the granddaughter's dog got really sick and died.

The granddaughter wrote her Nanna accompanied with a really sad picture of herself: "I really miss Sam - really really!" Her granny $\backslash$ answered: "I have been thinking of her too, but she was very sick \& you wouldn't want her to suffer, would you?"

The electronic Magic Box mediated comfort and shared understanding, and the exchange of fun messages. 


\subsection{New Quality Goals}

While we were interested to group the sub-qualities to our existing quality goals, in order to substantiate the quality goals with our field data, we permitted new main quality goals to emerge, and hence allow changes to our overall goal model. As part of the method, in the event important activities or themes evolve for which we cannot find a home, we define new quality goals. Qualities emerging that we could not group with our existing quality goals were themes surrounding the technology use itself still being close connected to positive feelings - often explicitly described as fun. The new quality goal that emerged is build confidence, shown in Figure 5.

Learning: One important aspect was being able to continuously improve managing the technology. Some of the grandparents expressed it this way:

It is quite interesting to see where we started: "I didn't find photo, but here is the text". Next time I was able to send the text as well. It is a bit of fun [GP]

I guess I have to get into email now with some kicking and screaming - I am enough of a dinosaur. I think I am ready [GP].

When an empty box was sent: a kangaroo would jump out of the box. The families described they had a lot of fun when this function was discovered. One grandchild could not figure out how this had happened and could not get enough of the kangaroo magically jumping out of the box (see Figure 5b); the grandmother was proud that she was able to do something unexpected with the technology that links in with building up confidence.

Creativity: It was seen as a challenge, but worthwhile effort to get the material for a creative message together.

When I had an inspiration and took a photo then that was a bit of fun.

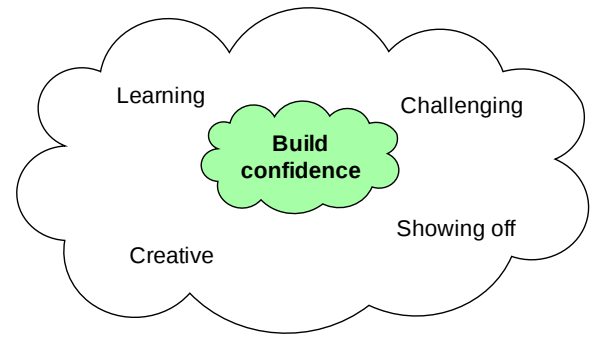

Fig. 5. New quality goal build confidence.

Mastering a challenge: Send a box off every day -an activity that is achievable, but still takes some effort such as to take a photo, "pack it" and send off in the box.

Magic box is sort of you are in Prep and then you are in grade one. When you start school you go up to grade one. Collage [the first technology probe we had introduced to that family] was Prep and electronic Magic Box is like grade one a step up. There is a little bit to it - to go into it. We graduated [GP].

I am sorry to see it go besides the fact that I don't have to wake up and think what should I send today? [GP]. 
The maze was not only fun for the children - here was where the children got their challenge as the technology was for them in most parts no problem to handle. Animal buttons for settings from easy (koala bear) to wicked (frog) were indicators for improvement. This challenge was important to the children without being competitive with others. It would engage them indirectly in an interaction with grandparents as they could play this game only when a message was sent by the grandparents and therefore was associated with the grandparents' interactions. In this regard the maze also fulfilled a balancing function for the fact that the children did not feel challenged by the technology. The quality goals are fulfilled for all users even though they can take different forms.

Showing off: Showing the application to people like neighbours, friends and other family members with a feeling of pride. This theme is a clear sign that confidence indeed had been built up and another example or measure for validating the success of the application that is closely tied to a complex quality goal and not to a certain piece of functionality. One mother said about her daughter:

Showing them something cool: 'this is what I've got this is mine' - this is my phone and I can send pictures [parent].

The showing off effect was in particular interesting with the grandparents. There was a new role the grandparents suddenly had among their peers. They became advocates for new technologies, while they would have never anticipated themselves as champions of new technologies. They found confidence in the technology that really had nothing to do with anything we had planned the technology to provide. However, in focussing on the grandparent-grandchild interaction and in keeping the technology lean and simple until the quality goals were better understood, we had catered for enjoyable use in a very substantial way (component 5).

\subsection{Understanding Socially-Oriented Requirements}

Figure 3 and Figure 5 show the substantiated quality goals after the ethnography was done (component 6). Newly formulated requirements are to a large extent influenced by the new quality cloud build confidence. Building confidence is part of the intergenerational interaction and it has implications on how the technology should be designed: not put everything in an application at once, because it scares the grandparents away. We now maintain simple screen views and a layered application instead of a packed one with functionality. Aiming for simplicity is not only based on the lack of confidence of many grandparents to deal with complex technology, but is suggested in the nature of strong-tie relationships themselves. It is apparent in the sub-themes that these technologies rely on an existing rich and loving relationship. To support the long-term interactions between grandparents and grandchildren, technologies need to mediate these subtle but complex relationships within the family context and routines. We cannot evaluate the success of the technology per se. We have to evaluate if the use of the technology supports the quality goals of the goal model. We use the model to see if it is indeed a representation of the socio-technical system (technology use of the grandparents with their grandchildren for the purpose of having fun). 
Another important insight was "the other side of fun". Certain familial values tend to be marginalised. For example, disclosing weaknesses or failure - and laughing about them - or the demonstration of grief and openly dealing with it, are not normally identified as laudable values in systems design. In our study, the grandmother does not try to brush the grief away with some happy comment, but she honestly acknowledges that the loss of the loved dog indeed is sad. Dealing with these kinds of emotions is just as important for a strong-tie relationship as demonstrating love, play together and laugh about a joke. It is no contradiction that technologies for intergenerational fun also allow and even aim for activities that deal with aspects we would normally avoid to show openly.

\subsection{Summary of Method for Modelling Social Interactions}

We have presented a method for substantiating quality goals in the development of domestic technologies to support interactions between grandparents and grandchildren. We used agent-based models for representing the goals and motivations of individuals with a focus on family values. We described six components as part of our approach. The components have allowed us to explore the trade-offs between functional richness and use of technology in the home. We use agent-oriented models to record the high-level goals and their quality attributes to represent social interactions, which can provide an account for social concepts such as fun or intimacy. The agent models proved to be particularly suitable to express culturally sensitive data obtained from field studies. As data gathered using probes is fragmented and unstructured, the process of translation from field data to the abstract generalisation required in development is difficult. The models provide a place where abstract design concepts can be collected and represented, helping the researchers and software engineers come to a shared understanding of the social domain.

\section{Conclusions}

In this chapter we have explored the use of agent-oriented models during system development in order to elicit, understand, and represent socio-cultural aspects of everyday life. In our case the domain of interest has been domestic technology use. A process for combining ethnographic data and agent-oriented models informs and substantiates understandings of the domain, family values and activities. The process generates findings that are often surprising, complex, and nuanced. The agentoriented models provide a place where abstract social activities and qualities can be collected and represented. They are a lens through which use activities can be analysed and recorded and then discussed amongst researchers and software engineers. A number of benefits emerged from our approach:

Sharing and making explicit. Fun, as many other social concepts, has many facets and it is beneficial to agree on a high-level view when building a socio-technical system. A shared view between software engineers and ethnographers helps to orient 
communication and focus the team on the relevant data during collection and analysis. There remains the ongoing possibility to change and refine this shared view during discussions. The motivational goal model allowed us to discover new quality goals whilst we learnt about new social aspects and attributes of the initial ones.

Grounding design in data. During development we were able to keep associations between the ethnographic data and the motivational models. We could understand the qualities of the clouds as examples of real social activities. These examples and associations were meaningful during the software engineering process when discussing high-level requirements for building new domestic technologies. With the agent-oriented models we were able to see the users' motivations made real in design.

Closing the gap and interleaving processes. There is a strong interplay and information exchange between the field data and the agent-oriented models. The standard software engineering process is a top down process. We used the high-level structured view -the quality goals - as a lens to analyse field data. Importantly, the fluid process influences the bottom-up information flow also. We changed the models as we discovered new qualities and learnt more about existing quality goals. In this sense we were matching two different perspectives, top-down and bottom-up. The suitability of our technologies demonstrates the extent to which the gap was closed and where we still had to achieve a better match between initial understanding and consequential implementation of the cultural model of intergenerational fun.

Traceability of motivations. This method is repeatable and traceable, as evidenced in statements such as "I made this decision because this was fun for so many families when". The possibility to refer to the context and trace motivations is a crucial process in the development of socio-technical systems.

Validation. With the quality clouds we were creating a set of new testing artefacts. They were useful in the process to validate associations between activities and highlevel goals and evaluate the degree of the match between the two. This took place in a participatory manner - including the grandparents and the grandchildren.

Overall the approach described above assisted ethnographers and software engineers in arriving at a shared understanding of social goals and the related interactions in a way that became useful in ongoing software development for the social domain.

Acknowledgments. We would like to thank the participating families. This research was funded by an Australian Research Council Discovery Grant (\#DP0880810).

\section{References}

1. Arnold, M. The connected home: probing the effects and affects of domesticated ICTs. In Proc. PDC 2004, ACM Press, 1-4. (2004)

2. Baxter G., and Sommerville, I.. Socio-technical systems: From design methods to systems engineering. Interacting with Computers. 23, 4-17. (2010) 
3. Bell, G., Blythe, M., and Sengers, P. Making by making strange: Defamiliarization and the design of domestic technologies. ACM Trans. Comput.-Hum. Interact. 12, 2, 149173. (2005)

4. Brown, B., Taylor A., Izadi S., Sellen A., Kaye J. J., and Eardley R. Locating Family Values: A Field Trial of the Whereabouts Clock. In Proc. UbiComp'07. 354-371 (2007).

5. Cao, X., Sellen, A., Brush, A. J. et al. Understanding family communication across time zones. In Proc. of CSCW 2010, ACM Press, 155-158. (2010)

6. Cattan, M., White, M., Bond, J., \& Learmouth, A. Preventing social isolation and loneliness among older people. Ageing \& Society, 25, 1, 41-67. (2005)

7. Chung LK, Nixon BA, Yu E, Mylopoulos J. Non-Functional Requirements in Software Engineering. Kluwer Publishing. (2000)

8. Dearden, A., and Rizvi, H. Participatory IT Design and Participatory Development: A Comparative Review. In Proc. PDC 2008, 1-10 (2008)

9. Druin, A., Bederson, B. B., and Quinn, A. Designing intergenerational mobile storytelling. In Proc. IDC 2009, ACM Press, 325-328. (2009)

10. Durrant, A., Taylor, A. S., Frohlich, D., Sellen, A., and Uzzell, D. Photo displays and intergenerational relationships in the family home. In Proc. BCS HCI 2009, ACM Press, 1019. (2009)

11. Garcia A. and N. Medinilla. The ambiguity criterion in software design. In International Workshop on Living with Uncertainties (IWLU'07). ACM, (2007)

12. Gause. D. User DRIVEN design the luxury that has become a necessity, a workshop in full life-cycle requirements management. In ICRE 2000, Tutorial T7, (2000)

13. Gaver B., Dunne T., and Pacenti E. Design: Cultural Probes. Interaction 6(1): 21-29. (1999)

14. Gibbs, M. Vetere F., Bunyan M., and Howard S. SynchroMate: A Phatic Technology for Mediating Intimacy. In Proc. DUX 2005, AIGA 37, 5 pages (2005)

15. Grivas, K. Digital Selves: Devices for intimate communications between homes. Personal Ubiquitous Comput. 10, 2-3, 66-76. (2006)

16. Gross H. Component-based Software Testing with UML. Springer. (2005)

17. Guizzardi R. and A. Perini. Analyzing requirements of knowledge management systems with the support of agent organizations. Journal of the Brazilian Computer Society (JBCS)-Special Issue on Agents Organizations, 11(1):51-62, (2005)

18. Harper, R. Inside the Smart Home: Ideas, possibilities, methods. In Harper, R. (Ed). Inside the Smart Home: Interdisciplinary perspectives on the design and shaping of domestic computing. Berlin Springer, 1-13. (2003)

19. Hassenzahl, M. The thing and I: Understanding the relationship between user and product. In M. Blythe, C. Overbeeke, A. Monk, P. Wright (eds.). Funology: From usability to enjoyment. Kluwer, 31-42. (2003)

20. Hemmings, T., Clarke, K., Crabtree, A., Rodden, T. and Rouncefield, M. Probing the probes. Domestic Probes and the Design Process. Proc. PDC 2002, 42-50. (2002)

21. Howard, S., Kjeldskov, J., and Skov, M. (eds). Pervasive Computing in the Domestic Space. J. Personal and Ubiquitous Computing, 329-414. (2006)

22. Hutchinson, H., Mackay, W., Westerlund, B., et al. Technology Probes: Inspiring Design for and with Families. In Proc. CHI 2003, ACM Press, 17-24. (2003)

23. Iqbal, R., James, J., and Gatward, R. Designing with ethnography: An integrative approach to CSCW design. Advanced Engineering Informatics, 19:81-92. (2005)

24. Judge, T. K., Neustaedter, C., and Kurtz, A. F. The family window: the design and evaluation of a domestic media space. In Proc. CHI 2010, ACM Press, 2361-2370. (2010)

25. Jureta I. J., and Faulkner S. Clarifying goal model. Conceptual modelling. Proc. ER, 139-144. (2007) 
26. Kirikova M, Grundspenkis J, Wojtkowski W, Zupancic J, and Wrycza S. Information Systems Development: Advances in Methodologies, Components, and Management. Springer. (2002)

27. Leonardi, C., Mennecozzi, C., Not, E., et al. Knocking on elders' door: investigating the functional and emotional geography of their domestic space. In Proc. CHI 2009, ACM Press, 1703-1712. (2009)

28. Lindley, S. E., Harper, R., and Sellen, A. Desiring to be in touch in a changing communications landscape: Attitudes of older adults. In Proc. SIGCHI 2009, ACM Press, 1693$1702(2009 a)$

29. Lindley, S. E., Sellen, A., and Harper R. Bridging the gap between grandparents and teenagers. CHI 2009b http://research.microsoft.com/en-us/groups/sds/ publications.aspx

30. Martin D, Sommerville I. Patterns of Cooperative Interaction: Linking Ethnomethodology and Design. ACM Transactions on Computer-Human Interaction 11(1):59-89. (2004)

31. Paay, J., Sterling, L., Vetere, F., Howard, S., and Boettcher, A. Engineering the Social: The Role of Shared Artifacts, IJHCS, 67(5):437-454. (2009)

32. Patton, M. Qualitative Research and Evaluation Methods. Sage Publications, Thousand Oaks California, (2002)

33. Pavon, J., Arroyo M., Hassan, S., and Sansores. C. Agent-based modelling and simulation for the analysis of social patterns. Pattern Recognition Letters, 29(8):1039-1048, 2008.

34. Pedell, S., Miller T., Vetere, F. et al. Having fun at home: interleaving fieldwork and goal models, Proc. OZCHI 2009, 309-312. (2009)

35. Petersen, M. G. Squeeze: designing for playful experiences among co-located people in homes. In Proc. CHI 2007, ACM Press, 2609-2614. (2007)

36. Reekie H., and McAdam R. A Software Architecture Primer: Angophora Press. (2006)

37. Sommerville, I. Software Engineering (8th ed). Addison Wesley, Harlow, Essex. (2007)

38. Sterling, L., and Taveter, K. The Art of Agent-Oriented Modelling. MIT Press. (2009)

39. Vetere, F., Gibbs, M., Kjeldskov, J., Howard, S., Mueller, F., Pedell, S., Mecoles, K., and Bunyan, M. Mediating Intimacy: Designing Technologies to Support Strong-Tie Relationships. Proc. CHI 2005, ACM, 471-480. (2005)

40. Vetere, F., Smith, J. and Gibbs, M. Phatic Interactions: Being Aware and Feeling Connected. In P. Markopoulos, B. de Ruyter, and W. Mackay (eds) Awareness Systems: Advances in Theory, Methodology and Design. Springer Verlag, London, 173-186. (2009)

41. Viller, S., and Sommerville, I. Ethnographically informed analysis for software engineers. IJHCS, 53(1):169-196. (2000)

42. Vutborg R., Kjeldskov J., Vetere F., and Pedell S. Family Storytelling for Grandparents and Grandchildren living apart. In Proc. NordiCHI 2010, ACM, 531-540. (2010)

43. Yarosh, S., Cuzzort, S., Müller, H. and Abowd, G. D. Developing a media space for remote synchronous parent-child interaction. In Proc. IDC 2009, ACM Press, 97-105. (2009)

44. Zafiroglu, A. and Chang, M. Digital homes on wheels: designing for the unimagined home. Personal Ubiquitous Comput. 11, 5, 395-402. (2007)

45. Randall, D., Harper, R., and Rouncefield, M. Fieldwork for Design: Theory and Practice. Springer. (2007) 